\title{
Drastic changes of the domain size in an ultrathin magnetic film
}

\author{
M. Kisielewski and A. Maziewski ${ }^{\mathrm{a})}$ \\ Institute of Experimental Physics, University of Bialystok, Lipowa 41, 15-424 Bialystok, Poland \\ V. Zablotskii \\ Institute of Physics ASCR, Na Slovance 2, 18221 Prague 8, Czech Republic \\ and Donetsk National University 83055, Donetsk, Ukraine \\ T. Polyakova \\ Donetsk National University 83055, Donetsk, Ukraine
}

J. M. Garcia

Laboratoire de Physique des Solides, Universite Paris-Sud and CNRS, Orsay, France

\author{
A. Wawro and L. T. Baczewski \\ Institute of Physics Polish Academy of Sciences, Al. Lotnikow 32/46, 02-668 Warsaw, Poland
}

(Presented on 12 November 2002)

\begin{abstract}
A general framework for the domain size in any ultrathin film with perpendicular magnetic anisotropy is here discussed. The domain structure is analyzed by using the classical theory taking into consideration the demagnetization field contribution to the domain wall energy. A sinusoidal model is considered to describe the domain structure while approaching, in two different cases, the monodomain state with in-plane magnetization. The first case is realized applying a large enough in-plane magnetic field. The second one is obtained by decreasing the perpendicular magnetic anisotropy, which is connected in many ultrathin systems with the increase of film thickness. A change in the domain size of several orders of magnitude is obtained while approaching the magnetization reorientation region. The minimal stripe domain period $p=8 \pi \ell_{\mathrm{ex}}^{2} / d$ is calculated from the sinusoidal model, where $\ell_{\mathrm{ex}}$ is the exchange length and $d$ is the thickness of the film. The range of possible domain size changes in ultrathin films is predicted. The domain size has been experimentally studied in a $1 \mathrm{~nm}$ Co film characterized by a square hysteresis loop. The investigations have been performed by polar Kerr based microscopy and magnetic force microscopy. The domain structure of two remnant states generated by applying an in-plane and a perpendicular magnetic field has been compared. Drastically, the smallest domain size has been observed for the former. (C) 2003 American Institute of Physics. [DOI: 10.1063/1.1556161]
\end{abstract}

Understanding the magnetic ordering of ultrathin films is of the utmost importance, not only for basic science but also for possible applications. A considerable number of experimental and theoretical works have been devoted to the analysis of domain structure (DS) properties in ultrathin magnets with different quality factors $Q\left(=K_{1} / 2 \pi M_{S}^{2}\right.$-the relation of the uniaxial anisotropy to the demagnetization energy) and different thickness, $d$. One can expect huge domain sizes in an ultrathin sample. ${ }^{1}$ However, very small domains were observed when approaching the reorientation phase transition (RPT) where a thickness increase induces a change from vertical to in-plane magnetization. ${ }^{2}$ The DS properties have been already studied in ultrathin cobalt films near the RPT induced by in-plane magnetic field, where very small domains, not available for visualization by optical microscopy, were expected. ${ }^{3}$

The present work is focused on both the theoretical and the experimental studies of equilibrium domain sizes range in an ultrathin magnetic film, characterized by thickness $d$, quality factor $Q$ and the exchange length

\footnotetext{
${ }^{a)}$ Electronic mail: magnet@uwb.edu.pl
}

$\ell_{\mathrm{ex}}=\left[\left(A /\left(2 \pi M_{S}^{2}\right)\right]^{0.5}\right.$ where $A$ is the exchange constant. The influence of an in-plane magnetic field $H_{\|}$is also here considered.

The DS has been studied in an ultrathin cobalt film prepared in a molecular beam epitaxy system. A sapphire single crystal wafer was covered by: (i) $20 \mathrm{~nm}$ Mo buffer layer grown at $1000{ }^{\circ} \mathrm{C}$; (ii) $20 \mathrm{~nm}$ Au deposited at room temperature; (iii) $1 \mathrm{~nm}$ Co layer; (iv) $8 \mathrm{~nm}$ Au coverage. Magnetooptical magnetometry revealed the effective magnetic anisotropy constant $\mathrm{K}_{1 \mathrm{eff}}=(0.473 \pm 0.003) \mathrm{MJ} / \mathrm{m}^{3}$. The measured square hysteresis exhibited $H_{c}=0.39 \mathrm{kOe}$. For ultrathin cobalt films the thickness dependence of $K_{1 \text { eff }}(d)$ is described by the well known formula: $K_{1 \text { eff }}=K_{1}-2 \pi M_{S}^{2}=K_{1 V}-2 \pi M_{S}^{2}$ $+2 K_{1 S} / d$ taking into consideration the volume and the surface contributions ${ }^{4}$ ( $K_{1 V}$ and $K_{1 S}$, respectively). Such parameters, $K_{1 V}$ and $K_{1 S}$, were determined for a Au/Co wedge/Au sample deposited on mica ${ }^{5}$ and used for the DS period analysis at a wide thickness range. The thickness of the phase reorientation transition for such ultrathin layer is typically in the range $1.7-2.0 \mathrm{~nm}$.

The DS has been visualized by an optical microscopy based on polar Kerr effect and magnetic force microscope (MFM). 
First, let us analyze the DS and its period in an uniaxial film with easy axis perpendicular to the plane submitted to $H_{\|}$field. The total sample energy, $E$, is described by the sum of the exchange, anisotropy, Zeeman and demagnetizing energies

$$
\begin{aligned}
E= & L_{x} d \int_{0}^{p / 2}\left(A\left(\frac{d \theta(y)}{d y}\right)^{2}+K_{1} \sin ^{2}(\theta(y))\right. \\
& \left.-M_{S} H_{\|} \sin (\theta(y))\right) d y+E_{D},
\end{aligned}
$$

where $L_{x}$ is the domain length along domain walls; $\theta(y)$ is the polar angle (a periodical function, with period $p$, determining the magnetization distribution in the film); $E_{D}$ describes the demagnetizing energy. Usually the demagnetizing energy term is evaluated without considering the domain wall width. We focus our discussion on two approaches: (i) assuming the demagnetization effect in the domain walls according to Schlömann's theory, ${ }^{6}$ (ii) considering a sinusoidal domain structure as the limiting DS at the reorientation region between two states of multi-domains and in-plane mono domain.

The zero-field case is well described by the classical theory ${ }^{7-9}$ for large enough $Q$ and negligible wall thickness. The equilibrium normalized period, $p_{0} / \ell_{c}$, as a function of the normalized thickness, $d / \ell_{c}$, can be described by a transcendent equation with the Lerch's functions, ${ }^{10}$ where $\ell_{c}$ is the characteristic length $\left[\left(\ell_{c}=\sigma_{w} /\left(4 \pi M_{z}^{2}\right)\right.\right.$ describes the ratio between the domain wall $\sigma_{w}$ and demagnetizing energies, $M_{z}$ being the $z$ component of the magnetization vector].

In this section we use the classical description to calculate $p(d)$-dependencies. The main problem is how to take into account the demagnetizing effect in domain wall energy for the ultrathin regime. Usually one considers, in the simplest approximation, that the wall energy is equal to $4\left(A K_{1 \text { eff }}\right)^{0.5}$. Under such assumption the domain period $p_{\text {eff }}$ drastically decreases when approaching the RPT thickness $d_{1}\left(K_{1 \text { eff }}=0\right)$. More carefully, the demagnetizing effect in the ultrathin regime when $d \ll \delta$ ( $\delta$ is the domain wall width) can be considered using $\sigma_{w}=\sigma_{w 0}-2 \pi M_{S}^{2} \delta$. For very thin films, i.e., in the limit $d \rightarrow 0$, the wall width depends on the quality factor $\mathrm{as}^{5} \quad \delta=\delta_{0}(1-1 / Q)^{-1 / 2} \quad$ (where $\delta_{0}=\pi\left[A /\left(K_{1 \text { eff }}\right.\right.$ $\left.\left.+2 \pi M_{S}^{2}\right)\right]^{0.5}$ is the wall width and $\sigma_{w 0}=4\left[A /\left(K_{1 \text { eff }}\right.\right.$ $\left.\left.\left.+2 \pi M_{S}^{2}\right)\right]^{0.5}\right)$. Then, from these expressions one can calculate $\sigma_{w}=\sigma_{w 0}[1-(1 / 4 Q \sqrt{1-1 / Q})]$. The domain period $p_{d w}$, calculated using this more precise approximation, drastically decreases approaching $d^{*}\left(<d_{1}\right)$ when $\sigma_{w}$ goes to zero. Comparing $p_{\text {eff }}$ and $p_{d w}$ one can find a significant influence of the demagnetizing contribution on the domain size. Figure 1(b) shows $p_{d w}\left(d / \ell_{\mathrm{ex}}\right)$ dependencies calculated for films with thickness independent magnetic anisotropy. Such materials are, for instance, the tetragonal ordered alloys studied in Ref. 11.

Let us now consider a domain structure near the RPT in the simple sinusoidal approximation where $\Theta(y)$ $=\Theta_{0} \sin (2 \pi y / p)(\Theta=\pi / 2-\theta)$, initially proposed for a semiinfinite magnetic space. ${ }^{12}$ Using the expression for $\Theta(y)$ in Eq. (1), the normalized total energy density is given by
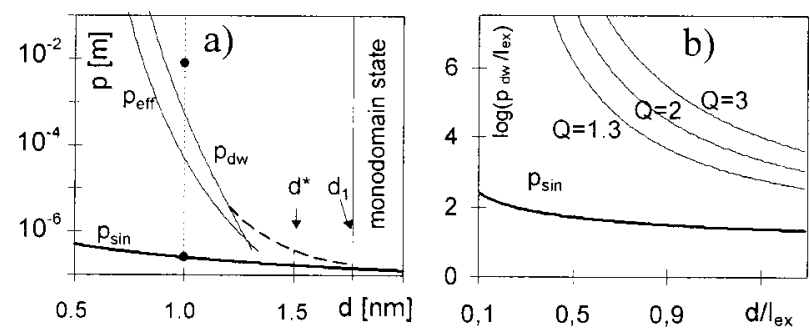

FIG. 1. Thickness dependence of the domain structure period. (a) Thin lines $-p_{\text {eff }}(d), p_{d w}(d)$ calculated from the classical model using $K_{1 V}$ $=0.85 \mathrm{MJ} / \mathrm{m}^{3}, K_{1 S}=0.37 \mathrm{~mJ} / \mathrm{m}^{2}$, as experimentally determined for ultrathin cobalt wedge (see Ref. 5) $\left(M_{S}=1420 \mathrm{Gs}\right.$ and $\ell_{\mathrm{ex}}=3.2 \mathrm{~nm}$ were assumed); $d^{*}$ and $d_{1}$ are also determined for these parameters. The thick line describes $p_{\text {sin }}(d)$ calculated from Eq. (3) showing the in-plane magnetic field induced DS size. The dashed line connects the $p_{d w}(d)$ and the $p_{\text {sin }}\left(d_{1}\right)$ in the thickness region where the classical theory does not work. The dotted line represents the stripe domain period region available for the investigated 1-nmthick Co sample. (b) Thin lines- $p_{d w}(d)$ calculated from the classical model for films with different thickness independent $Q$ factor. The thick line describes $p_{\sin }(d)$.

$$
\begin{aligned}
\frac{E_{\mathrm{sin}}}{2 \pi M_{S}^{2} \ell_{\mathrm{ex}}}= & \frac{d}{\ell_{\mathrm{ex}}}\left(\frac{2 \ell_{\mathrm{ex}}^{2} \pi^{2} \Theta_{0}^{2}}{p^{2}}+Q-\frac{\Theta_{0}^{2}}{2} Q-2 \frac{H_{\|}}{4 \pi M_{S}}\right. \\
& \left.\times\left(1-\frac{\Theta_{0}^{2}}{4}\right)\right)+\frac{p \Theta_{0}^{2}}{4 \pi \ell_{e x}}\left(1-\operatorname{Exp}\left(-\frac{2 \pi d}{p}\right)\right) .
\end{aligned}
$$

Minimizing Eq. (2) with respect to $p$ and assuming $d / p \ll 1$, one can find this simple formula for the equilibrium period

$$
p_{\mathrm{sin}}=\frac{8 \pi \ell_{\mathrm{ex}}^{2}}{d} .
$$

It is worth noting that the $p_{\text {sin }}$ period does not depend on the anisotropy constants and it is the minimal possible domain period, which can be achieved for any sample applying large enough $H_{\|} \cdot{ }^{13}$ The period is much smaller (see Fig. 1) than the equilibrium one given by the classical model. The minimal period can be also reached approaching the RPT by the thickness change.

The question is: what is the equilibrium domain period in the thickness range $d<d_{1}$ for a given set of material parameters $K_{1 S}, K_{1 V}, M_{S}$ and $\ell_{\text {ex }}$ ? There are two curves $p_{\text {eff }}(d), p_{d w}(d)$ and the point for the minimal period $p_{\text {sin }}$ available for the defined material parameters at thickness near $d_{1}$, see Fig. 1(a). One can deduce that the classical model cannot be applied for a significant thickness region near the RPT where a more complicated magnetization distribution should be considered, see, e.g., the model given in Refs. 11 and 14. We have extrapolated the $p_{d w}(d)$ to the $p_{\sin }\left(d_{1}\right)$ point by the dashed line.

It is possible to have different domain periods $p_{\sin } \leqslant p$ $\leqslant \infty$, in a sample with a given thickness, depending on the sample parameters (e.g., coercivity force) and its magnetic history. In order to give an experimental example, we have studied an ultrathin Co film (1 nm thick). We have showed in Fig. 1 the range of available periods for this sample. Using the classical and the sinusoidal models discussed above, one can find a difference of more than four orders of magnitude 


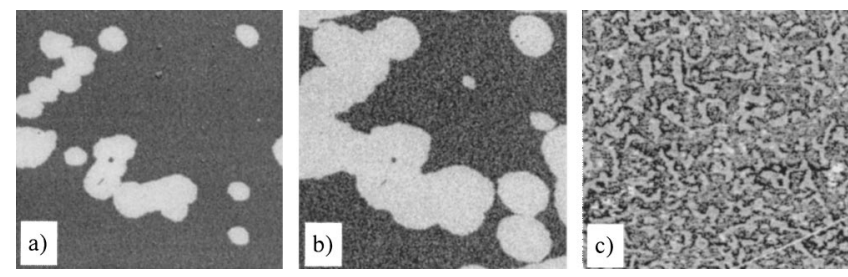

FIG. 2. Remnant domain structures visualized using: (a) and (b) magnetooptical microscope (image size $2 \mathrm{~mm} \times 2 \mathrm{~mm}$ ); these images were obtained after the first and second magnetic field pulses (magnitude 382 Oe and time duration $1 \mathrm{~s}$ ), respectively; (c) magnetic force microscope (image size 20 $\mu \mathrm{m} \times 20 \mu \mathrm{m})$; the structure was produced by in-plane magnetic field.

in two equilibrium periods determined for: (i) the zero field state, $p_{d w}=8.3 \mathrm{~mm}$; (ii) the state near the $H_{\|}$-induced RPT, $p_{\text {sin }}=258 \mathrm{~nm}$, Fig. 1 .

We have produced different domain structures by using two methods: (i) applying a perpendicular magnetic field along one direction ("white" in the image) in a sample previously saturated along the opposite ("black") direction; (ii) switching off a large enough $H_{\|}$which previously saturated the sample. The results are shown in Figs. 2(a) and 2(b) (magneto-optical image) and Fig. 2(c) (MFM image), respectively.

The high coercivity prevents the system from reaching the equilibrium state. Let us consider the remagnetization process [shown in Figs. 2(a) and 2(b)], which has been started from saturated sample. Because of the small magnetostatic force in comparison to the coercivity, the increase of white domains goes through an almost isotropic expansion from the nucleation centers. This process is quite different from that observed in, e.g., bubble materials ${ }^{9}$ where such expansion proceeds by the increase of volume of curved stripes with rather well defined width determined by the magnetostatic and wall energies.

The domain pattern produced with an in-plane field is completely different than that obtained with a perpendicular one. The pattern from Fig. 2(c) is similar to the DS observed for bubble materials, e.g., garnets. ${ }^{9}$ The formation of domains from the saturated state occurs by nucleation in many centers and by branching of curved stripes with about 0.6 $\mu \mathrm{m}$ width. The width is of the same order as $p_{\sin }$. So, the fine DS was formed as the equilibrium one at the $H_{\|}$induced RPT. Due to the coercivity force, the structure is "frozen" after switching off the field.

The authors are grateful to Professor J. Miltat for fruitful discussions concerning MFM imaging and Dr. M. Tekielak for magneto-optical imaging. J. M. Garcia wishes to thank the European Community for his Marie Curie Fellowship. This work was supported by the Polish State Committee for Scientific Research (Grant No. 4 T08A 025 23), ESF NANOMAG project and European Commission program ICA1-CT-2000-70018 (Center of Excellence CELDIS).

${ }^{1}$ B. Kaplan and G. A. Gehring, J. Magn. Magn. Mater. 128, 111 (1993).

${ }^{2}$ M. Speckmann, H. P. Oepen, and H. Ibach, Phys. Rev. Lett. 75, 2035 (1995).

${ }^{3}$ A. Stankiewicz, A. Maziewski, J. Ferre, and V. Grolier, Appl. Phys. Lett. 74, 2519 (1999).

${ }^{4}$ Ultrathin Magnetic Structures, edited by B. Heinrich and J. A. C. Bland (Springer, Berlin, 1994), and references therein.

${ }^{5}$ M. Kisielewski, Z. Kurant, A. Maziewski, M. Tekielak, N. Spiridis, and J. Korecki, Phys. Status Solidi 189, 3 (2002).

${ }^{6}$ E. Schlömann, J. Appl. Phys. 44, 1837 (1973).

${ }^{7}$ C. Kooy and U. Enz, Philips Res. Rep. 15, 7 (1960).

${ }^{8}$ Z. Malek and V. Kambersky, Czech. J. Phys. 8, 416 (1958).

${ }^{9}$ A. Hubert and R. Schäfer, Magnetic Domains (Springer, Berlin, 1998).

${ }^{10}$ A. Maziewski, V. Zablotskii, and M. Kisielewski, Phys. Status Solidi A 189, 1001 (2002).

${ }^{11}$ Y. Samson, A. Marty, R. Hoffmann, V. Gehanno, and B. Gilles, J. Appl. Phys. 85, 4604 (1999); A. Marty, Y. Samson, B. Gilles, M. Belakhovsky, E. Dudzik, H. Durr, S. S. Dhesi, G. van der Laan, and J. B. Goedkoop, ibid. 87, 5472 (2000).

${ }^{12}$ N. Saiti, H. Fujiwara, and Y. Sugita, J. Phys. Soc. Jpn. 19, 1116 (1964).

${ }^{13}$ More detailed discussion of domain structure is prepared for publication.

${ }^{14}$ A. L. Sukstanskii and K. I. Primak, J. Magn. Magn. Mater. 169, 31 (1997). 